\title{
$1 / N$ corrections to the binding energy of an Anderson model fluctuating between two magnetic configurations
}

\author{
J M Landgraf and J W Rasul \\ The Harrison M Randall Laboratory of Physics, University of Michigan, Ann Arbor, \\ MI 48109-1120, USA
}

Received 17 June 1993, in final form 16 August 1993

\begin{abstract}
We investigate the binding energy separation between the many-body ground-state singlet and higher-lying magnetic states for the $\mathrm{f}^{1}-\mathrm{f}^{2}$ Anderson model to next-leading order in the $1 / N$ expansion, where $N$ denotes the orbital degeneracy of the $\mathrm{f}$ states. We formulate integral equations for the magnetic-state amplitudes that appear at next-leading order in $1 / \mathrm{N}$ and solve these, together with the integral equations that describe the singlet, numerically. We find that the total separation between magnetic and singlet states is small, even in the valencefluctuation regime, and hence demonstrate the robustness of the leading-order result. We find that the maximum binding-energy separation is shifted towards higher valence, in contradiction to results of the minimal-degeneracy model. We also present results for the valence and charge susceptibility.
\end{abstract}

\section{Introduction}

Among the many aspects of correlated electron systems that attract current attention, the heavy-fermion state in certain rare-earth and actinide systems continues to perplex investigators. In spite of the progress made in understanding the single-impurity problem, there are, even without including intersite effects, still features of the extreme dilute alloys that require clarification. In particular the extension of the Anderson model to the case where both of the two lowest-lying configurations possess magnetic moments provides a particular challenge. The majority of the early work on this problem has been concerned with the case where the conduction electrons have only a spin- $\frac{1}{2}$ degeneracy [1] while the magnetic atomic configurations have a greater number of internal degrees of freedom. Numerical renormalization group [2] and Bethe ansatz [3] solutions of this problem show that the ground state possesses a magnetic moment (see [4] for a review).

At present much attention is being focused on the 'opposite' limit, where the conduction electrons have more internal degrees of freedom than the local moments, and an 'overscreened' ground state, with power-law dependences of the bulk properties on temperature, is the result. Such a situation arises when the $\mathrm{f}^{2}$ state is split by crystal fields so that a non-magnetic doublet is obtained, which then undergoes quadrupolar charge fluctuations into the higher-lying $\Gamma_{6}$ state [5]. One system that shows such non-Fermiliquid-like behaviour is $\mathrm{UYPd}_{3}[6]$.

At the same time a variety of models exist in which electron and impurity states of the same symmetry mix, and in this case the $L-S$ or $j-j$ coupling scheme is more appropriate [7]. A highly idealized model is to take the $j-j$ coupling scheme, but without any actual $j-j$ coupling [8]. This model then consists of $(N=2 j+1)$-fold degenerate local states (either singly or doubly occupied) mixing with an $N$-fold degenerate electron band. The advantages 
of this model are that it allows an expansion of ground-state properties in powers of $1 / N$ and allows a systematic study of the effects of successively adding electron-hole pair processes. Even if $N$ is not large, the issue is the extent to which this treatment captures the essential physics of the true finite- $N$ ground state, and provides an appropriate way of reordering perturbation theory so as to avoid unphysical low-energy divergences. At the same time it should be cautioned that for more realistic coupling schemes this model might not be appropriate, especially if a different ground-state symmetry is involved. Additionally, while the leading-order results of this model are independent of whether $L-S$ or $j-j$ coupling is used, the effective degeneracy is less for $L-S$ coupling, which may imply that more orders of $1 / N$ are necessary. Nonetheless, this $1 / N$ expansion scheme is implementable and its consequences should be explored.

Previous studies $[7,8,10,12]$ have shown that this system shows Kondo-like behaviour not only in the integer-valent limits, but throughout its valence range, and shows the existence of a small energy scale separating the singlet many-body ground state from higherlying magnetic states. This is to be contrasted with the $\mathrm{f}^{0}-\mathrm{f}^{1}$ problem where in the valence fluctuation range, the relevant energy scale separating the ground and excited states is of the order of the hybridization width. In addition, for the $\mathrm{f}^{1}-\mathrm{f}^{2}$ problem, the charge susceptibility, defined as the rate of change of the valence with bare $\mathbf{f}^{1}-\mathrm{f}^{2}$ level separation is found to be extremely small [10].

While these earlier studies are illuminating, they have been restricted in their range of applicability, being either variational $[7,9,10]$, non-crossing approximation [12] or leadingorder $1 / N$ expansion [8]. There has been an exact numerical renormalization group study [11] of the case with a $J=\frac{3}{2} \mathrm{f}^{\mathrm{h}}$ state and a $J=2 \mathrm{f}^{2}$ state which confirms the existence of a singlet ground state with a low-energy scale defining the low-temperature behaviour. However from a practical point of view, it is important to see how for the more approximate, but many-body standpoint, more readily implementable methods can provide a reasonable description of the ground-state features. Systematic corrections to these leading-order studies have not been explicitly evaluated, although the low-temperature extension to next order in $1 / N$ has been formulated by one of the authors [13] and one of the earlier variational studies [10] did include an extra electron-hole term in the $f^{1}$ component of the wavefunction. The question naturally arises as to whether the $1 / N$ corrections verify the robustness of this small energy scale found in the early studies. It was found that in the $\mathrm{f}^{0}-\mathrm{f}^{1}$ cerium Anderson model that although for most of the valence range the $1 / N$ corrections yielded small changes in universal features, such as the susceptibility versus valence, qualitative changes were found in the integer-valent regime [14].

From a physical point of yiew it is important whether this small energy scale survives higher-order corrections since the energy separation between singlet and higher-lying magnetic states indicates how strong intersite magnetic interactions have to be in order to induce magnetic ordering. This is of particular relevance not only for HF systems but also from the point of view of the low magnetic ordering temperatures in uranium rocksalt systems. In this paper we calculate the $1 / N$ corrections to the singlet-magnetic-state energy separation in the ground state. The integral equations determining the singlet energy have been derived elsewhere by one of the present authors [13], but they are unwieldy, involving solving integral equations for quantities that are functions of three continuous energy variables, and their numerical solution is very involved. In addition we formulate the theory for the magnetic states at zero temperature, which also require the solution of a set of integral equations. We shall carry this procedure out in terms of the reduced-basis diagonalization procedure of Gunnarsson and Schönhammer [15]. This yields systematic corrections in $1 / N$ to the magnetic-state energy. 
In the following section we derive the integral equation for a general set of possible magnetic states in the $\mathrm{f}^{1}-\mathrm{f}^{2}$ Anderson model. We also recap the singlet-state equations and sketch a simple derivation of these. We analyse these equations with respect to their important low-energy features and extract the singular behaviour, leaving a set of integral equations that are solved. In the final section we present the numerical results for the singlet- and magnetic-state energies, the singlet-magnetic separation, the valence and the charge susceptibility as a function of ionic separation or valence. We compare the behaviour of the solutions with the asymptotic solutions of the integral equations, and compare the results for the singlet-magnetic energy separation with results of earlier perturbative scaling theory [8]. We also compare this singlet-magnetic energy separation with earlier variational theories [7,10], and compare the effects associated with the $1 / N$ corrections on universal features, such as the singlet-magnetic separation as a function of valence.

Our starting point is the $\mathrm{f}^{1}-\mathrm{f}^{2}$ Anderson impurity model, which is given in a $j-j$ coupling scheme in the limit of zero $j-j$ coupling [8]:

$$
H=\sum_{k, m} \epsilon_{k m} c_{k m}^{\dagger} c_{k m}+V \sum_{k, m}\left(|m\rangle c_{k m}+c_{k m}^{\dagger}\langle m|\right)+E_{1} \sum_{m}|m\rangle\left\langle m\left|+E_{2} \sum_{m<m^{\prime}}\right| m m^{\prime}\right\rangle\left\langle m m^{\prime}\right|
$$

where the $c_{k m}^{\dagger}$ represent electron creation operators in a state labelled by the momentum $k$ and combined spin and angular momentum index $m$, with the bare energy $\epsilon_{\mathrm{km}}$. The local states are represented by states $\left[m\right.$ ) with bare energies $E_{1}$ and $E_{2}$ for the $\mathrm{f}^{1}$ and $\mathrm{f}^{2}$ states, respectively. The hybridization mixing-matrix element $V$ is taken to be independent of $k$ and $m$.

\section{Formulation of the integral equations}

In this section we derive the integral equations necessary to describe the energies of the magnetic and singlet states using the reduced-basis diagonalization procedure of Gunnarsson and Schönhammer [15].

\subsection{Magnetic states}

We will give the derivation of the magnetic states using as the starting point the doublet (or $n$-tuplet) state $|\mu\rangle=f_{\mu}^{\dagger}|0\rangle$, where $|0\rangle$ denotes the filled conduction-electron Fermi sea. We will show later that more general descriptions of these states reduce essentially to the same set of eigenvalue equations. The $n$-tuplet couples to the states

$$
|\epsilon \mu\rangle=\frac{1}{\sqrt{N-1}} \sum_{\nu, v \neq \mu} c_{\epsilon \nu} f_{\nu}^{\dagger} f_{\mu}^{\dagger}|0\rangle
$$

which have two electrons in the $\mathrm{f}^{2}$ states, one of which is coupled to a bare hole of wavevector $k$ and the other which carries angular momentum $\mu$. At leading order in $1 / N$ the total wavefunction is

$$
|\psi\rangle=\alpha_{0}|\mu\rangle+\sum_{\epsilon} \beta_{0}(\epsilon)|\epsilon \mu\rangle
$$


Substituting into the Schrödinger equation leads to the coupled set of equations

$$
\begin{aligned}
& \alpha_{0} \epsilon_{10}^{\mathrm{m}}+V \sqrt{N-1} \sum_{\epsilon} \beta_{0}(\epsilon)=0 \\
& \alpha_{0} V \sqrt{N-1}+\left(\epsilon_{20}^{\mathrm{m}}-\epsilon\right) \beta_{0}(\epsilon)=0
\end{aligned}
$$

where $\epsilon_{10}^{\mathrm{m}}=E_{1}-E_{0}^{\mathrm{m}}, \epsilon_{20}^{\mathrm{m}}=E_{2}-E_{0}^{\mathrm{m}}$ denote the leading-order separations between the magnetic-state energy $E_{0}^{\mathrm{m}}$ and the bare f-state energies. Eliminating $\beta_{0}(\epsilon)$ leads to the simple transcendental equation

$$
\epsilon_{10}^{\mathrm{m}}=\Gamma \log \left[\left(\epsilon_{20}^{\mathrm{m}}+D\right) / \epsilon_{20}^{\mathrm{m}}\right]
$$

where $\Gamma=\rho V^{2}(N-1)$, and $D$ is the bandwidth. In the $\mathrm{f}^{1}$ limit $\left(E_{2}-E_{2} \gg \Gamma\right)$ equation (5) has a binding energy $\epsilon_{10}^{\mathrm{m}}=\Gamma \log \left[\left(E_{21}+D\right) / E_{21}\right]$, and in the $\mathrm{f}^{2}$ limit has a binding energy $\epsilon_{20}^{\mathrm{m}}=T_{\mathrm{K}}=D \exp \left(-\left|E_{21}\right| / \Gamma\right)$. In the latter case this corresponds to a partial Kondo compensation of one of the stable moments, while in the former it yields only residual valence fluctuation corrections.

At the $1 / N$ correction level we must include first the $\mathrm{f}^{1}$ states

$$
\begin{aligned}
& |E, \epsilon, \mu\rangle=\frac{1}{\sqrt{N-1}} \sum_{\nu, \nu \neq \mu} c_{E \nu}^{\dagger} c_{\epsilon \nu} f_{\mu}^{\dagger}|0\rangle \\
& |E, \mu, \epsilon\rangle=\frac{1}{\sqrt{N-1}} \sum_{\nu, \nu \neq \mu} c_{E_{\mu}}^{\dagger} c_{\epsilon \nu} f_{\nu}^{\dagger}|0\rangle
\end{aligned}
$$

where $E$ is taken to be above the Fermi energy and $\epsilon$ is taken below the Fermi energy. In the first state the moment is carried by the felectron while in the second state it is carried by the conduction electron.

These couple to the $f^{2}$ states

$$
\begin{aligned}
& \left|E, \epsilon, \epsilon^{\prime}, \mu\right\rangle=\frac{1}{\sqrt{(N-1)(N-2)}} \sum_{\nu, \nu^{\prime}, \nu \neq \nu^{\prime} \neq \mu} c_{E \nu}^{\dagger} c_{\epsilon \nu} c_{\epsilon^{\prime} \nu^{\prime}} f_{\nu^{\prime}}^{\dagger} f_{\mu}^{\dagger}|0\rangle \\
& \left|E, \mu, \epsilon, \epsilon^{\prime}\right\rangle=\frac{1}{\sqrt{(N-1)(N-2)}} \sum_{\nu, \nu^{\prime}, \nu \neq \nu^{\prime} \neq \mu} c_{E \mu}^{\dagger} c_{\epsilon \nu} f_{\nu}^{\dagger} c_{\epsilon^{\prime} \nu^{\prime}} f_{\nu^{\prime}}^{\dagger}|0\rangle
\end{aligned}
$$

which only differ from (6) in having an extra electron-hole-impurity operator pair. Again, in the former the moment is carried by the uncompensated impurity, while in the latter the moment is carried by the conduction electron. Note that in $\left|E, \mu, \epsilon, \epsilon^{\prime}\right\rangle$ the ordering of $\epsilon$ and $\epsilon^{\prime}$ has no effect. Thus, to avoid double counting of the states we restrict the energies such that $\epsilon\left\langle\epsilon^{\prime}\right.$. This consideration does not affect $\left.\mid E, \epsilon, \epsilon^{\prime}, \mu\right\rangle$.

In terms of these states the $1 / N$ magnetic wavefunction takes the form

$$
\begin{aligned}
\left|\psi_{\mathrm{m}}\right\rangle=\alpha|\mu\rangle+ & \left.\sum_{\epsilon} \beta(\epsilon) \mid \epsilon, \mu\right\}+\sum_{\epsilon, E} \gamma_{1}(E, \epsilon)|E, \epsilon, \mu\rangle+\sum_{\epsilon, E} \gamma_{2}(E, \epsilon)|E, \mu, \epsilon\rangle \\
& +\sum_{\epsilon, \epsilon^{\prime}, E} \delta_{1}\left(E, \epsilon, \epsilon^{\prime}\right)\left|E, \epsilon, \epsilon^{\prime}, \mu\right\rangle+\sum_{\substack{\epsilon, \epsilon^{\prime}, E \\
\epsilon<\epsilon^{\prime}}} \delta_{2}\left(E, \epsilon, \epsilon^{\prime}\right)\left|E, \mu, \epsilon, \epsilon^{\prime}\right\rangle
\end{aligned}
$$


Applying the Schrödinger equation leads, after much algebra, to the following set of equations:

$$
\begin{aligned}
& \epsilon_{10}^{\mathrm{m}} \alpha+V \sqrt{N-1} \sum_{\epsilon} \beta(\epsilon)=0 \\
& \alpha V \sqrt{N-1}+\left(\epsilon_{20}^{\mathrm{m}}-\epsilon\right) \beta(\epsilon)+\sum_{E} V\left[\gamma_{1}(E, \epsilon)-\gamma_{2}(E, \epsilon)\right]=0 \\
& V \beta(\epsilon)+\left(\epsilon_{10}^{\mathrm{m}}-\epsilon+E\right) \gamma_{1}(E, \epsilon)+V \sqrt{N-2} \sum_{\epsilon^{\prime}} \delta_{1}\left(E, \epsilon, \epsilon^{\prime}\right)=0 \\
& V \sqrt{N-2} \gamma_{1}(E, \epsilon)+\left(\epsilon_{20}^{\mathrm{m}}-\epsilon+E-\epsilon^{\prime}\right) \delta_{1}\left(E, \epsilon, \epsilon^{\prime}\right)=0 \\
& -V \beta(\epsilon)+\left(\epsilon_{10}^{\mathrm{m}}-\epsilon+E\right) \gamma_{2}(E, \epsilon)+V \sqrt{N-2} \sum_{\epsilon^{\prime}} \delta_{2}\left(E, \epsilon, \epsilon^{\prime}\right)=0 \\
& V \sqrt{N-2}\left[\gamma_{2}(E, \epsilon)+\gamma_{2}\left(E, \epsilon^{\prime}\right)\right]+\left(\epsilon_{20}^{\mathrm{m}}-\epsilon+E-\epsilon^{\prime}\right) \delta_{2}\left(E, \epsilon, \epsilon^{\prime}\right)=0 .
\end{aligned}
$$

Here it is implied that sums over upper-case energies are for $E>0$ while sums over lowercase energies are for $\epsilon<0$. As before it is easy to eliminate the $f^{2}$ coefficients in terms of the $\mathrm{f}^{1}$ coefficients. It is traditional, for the leading-order theory, to scale $V$ and $N$ such that the combination $\Gamma=\rho V^{2} N$ is finite. In order for the $1 / N$ corrections to be meaningful we must demand that the $1 / N$ analysis gives the same results as the leading-order analysis when the $1 / N$ state amplitudes $\gamma$ and $\delta$ are set to zero. This forces us to take instead $\Gamma=\rho V^{2}(N-1)$. We then obtain the following equation for the magnetic binding energy correct to order $1 / N$ :

$$
\epsilon_{10}^{\mathrm{m}}=\Gamma \log \frac{\epsilon_{20}^{\mathrm{m}}+D}{\epsilon_{20}^{\mathrm{m}}}+\frac{\Gamma}{N} \int_{-D}^{0} \mathrm{~d} \epsilon \int_{0}^{D} \mathrm{~d} E \frac{Q_{1}(E, \epsilon)+Q_{2}(E, \epsilon)}{\epsilon_{20}^{\mathrm{m}}-\epsilon}
$$

where the functions $Q_{1}(E, \epsilon)$ and $Q_{2}(E, \epsilon)$ are proportional to $\gamma_{1}(E, \epsilon)$ and $\gamma_{2}(E, \epsilon)$ respectively, and satisfy the equations

$$
\begin{aligned}
& Q_{1}(E, \epsilon)=\frac{\Gamma}{d^{\mathrm{m}}(E-\epsilon)\left(\epsilon_{20}^{\mathrm{m}}-\epsilon\right)} \\
& d^{\mathrm{m}}(E-\epsilon) Q_{2}(E, \epsilon)=\frac{\Gamma}{\epsilon_{20}^{\mathrm{m}}-\epsilon}+\Gamma \int_{-D}^{0} \mathrm{~d} \epsilon^{\prime} \frac{Q_{2}\left(E, \epsilon^{\prime}\right)}{\epsilon_{20}^{\mathrm{m}}+E-\epsilon-\epsilon^{\prime}}
\end{aligned}
$$

where

$$
d^{\mathrm{m}}(E-\epsilon)=\epsilon_{10}^{\mathrm{m}}+E-\epsilon-\Gamma \log \left[\left(\epsilon_{20}^{\mathrm{m}}+E-\epsilon+D\right) /\left(\epsilon_{20}^{\mathrm{m}}+E-\epsilon\right)\right] .
$$

The fact that $Q_{2}(E, \epsilon) \propto \gamma_{2}(E, \epsilon)$ satisfies an integral equation reflects the fact that the angular momentum of the state $\{E, \mu, \epsilon\}$ is carried by the electron with energy $E$. This state couples to hole-impurity pairs of different energies as a result of the mixing into the $f^{2}$ state $\left|E, \mu, \epsilon, \epsilon^{\prime}\right\rangle$. As such, it parallels the development of both the leading and next-leading order in $1 / N$ singlet state.

Finally, expanding the right-hand side of $(10)$ to leading and next-leading order in $1 / N$ yields the following equation for the $1 / N$ correction to the magnetic-state binding energy $\delta E_{\text {mag }}$ :

$$
\left(1+\frac{\Gamma}{\epsilon_{20}^{\mathrm{m}}}-\frac{\Gamma}{\epsilon_{20}^{\mathrm{m}}+D}\right) \delta E_{\mathrm{mag}}=-\Gamma \int_{-D}^{0} \mathrm{~d} \epsilon \int_{0}^{D} \mathrm{~d} E \frac{Q_{1}(\epsilon, E)+Q_{2}(\epsilon, E)}{\epsilon_{20}^{\mathrm{m}}-\epsilon}
$$


where $\epsilon_{10}^{\mathrm{m}}$ and $\epsilon_{20}^{\mathrm{m}}$ are the leading-order results, $Q_{1}(\epsilon, E)$ and $Q_{2}(\epsilon, E)$ are given by (11) and (12), and $\delta E_{\text {mag }}$ is defined by the relation $E^{\mathrm{m}}=E_{0}^{\mathrm{m}}+(1 / N) \delta E_{\mathrm{mag}}$.

Similar magnetic states can, as remarked by Gunnarsson and Schönhammer [15], be written in the form

$$
\left|\epsilon^{(i)}\right\rangle=\sum_{\nu} C_{\nu}^{(i)} c_{\epsilon \nu} f_{\nu}^{\dagger}|0\rangle
$$

where $\sum_{\nu} C_{v}^{(i)}=0, \sum_{\nu} C_{\nu}^{(i)^{2}}=1$ (these states can carry a moment or not depending on the specific choice of the $C_{v}^{(i)}$ ). Despite the presence of the extra hole operator in $\left|\epsilon^{(i)}\right\rangle$ the equations obtained by acting on this state with $H$ are almost identical to the above formulation for $|\mu\rangle$. This can be easily seen from the results of acting on $\left|\epsilon^{(i)}\right\rangle$ twice with $H_{\operatorname{mix}}$ to leading order in $1 / N$.

$$
\begin{aligned}
H_{\text {mix }}\left|\epsilon^{(i)}\right\rangle= & \left.(V \sqrt{(N-1)}) \frac{1}{\sqrt{(N-1)}} \sum_{\substack{\epsilon, v_{1} \nu^{\prime} \\
\nu \neq \nu^{\prime}}} c_{\epsilon^{\prime} \nu^{\prime}} f_{\nu^{\prime}}^{\dagger} C_{\nu}^{(i)} c_{\epsilon \nu} f_{\nu}^{\dagger}|0\rangle \equiv V \sqrt{(N-1)} \mid \epsilon^{\prime}, \epsilon^{(i)}\right\} \\
H_{\text {mix }}\left|\epsilon^{\prime}, \epsilon^{(i)}\right\rangle & =V \sqrt{(N-1)} \sum_{\nu} C_{\nu}^{(i)} c_{\epsilon \nu} f_{\nu}^{\dagger}|0\rangle-\frac{V}{\sqrt{(N-1)}} \sum_{\epsilon^{\prime}, \nu} C_{\nu}^{(i)} c_{\epsilon^{\prime} \nu} f_{\nu}^{\dagger}|0\rangle \\
& =V \sqrt{(N-1)}\left|\epsilon^{(i)}\right\rangle-\frac{V}{\sqrt{(N-1)}} \sum_{\epsilon^{\prime}}\left|\epsilon^{\prime(i)}\right\rangle
\end{aligned}
$$

The second term on the right-hand side of $(16)$ is of the order of $1 / N$ as compared to the first term and may be neglected in the leading-order treatment. Thus, the $\mathrm{f}^{1}$ states $\left|\epsilon^{(i)}\right\rangle$ do not couple to $f^{1}$ states of other energies and the extra electron-hole operator in $\left|\epsilon^{(i)}\right\rangle$ only contributes an extra single-hole energy to the leading-order magnetic-state energy (5) at leading order. The extension of these results to the order of $1 / N$ and the effects of the second term on the right-hand side of (16) are described in the appendix where it is shown that the net effect of these terms is to raise the energies of the generalized magnetic states above those obtained from (8).

\subsection{Singlet state}

The integral equations derived earlier for the singlet 'quasiparticle energy' at finite temperature [13] can be readily derived using the Gunnarsson-Schönhammer reduced-basis method. The basis vectors we need to include are

$$
\begin{aligned}
& |\epsilon\rangle=\frac{1}{\sqrt{N}} \sum_{\nu} c_{\epsilon \nu} f_{\nu}^{\dagger}|0\rangle \\
& \left|\epsilon, \epsilon^{\prime}\right\rangle=\frac{1}{\sqrt{N(N-1)}} \sum_{\nu, \nu^{\prime}, \nu \neq \nu^{\prime}} c_{\epsilon^{\prime} \nu^{\prime}} f_{\nu^{\prime}}^{\dagger} c_{\epsilon \nu} f_{\nu}^{\dagger}|0\rangle
\end{aligned}
$$

which are, at the leading order in $1 / N$, states corresponding to one and two $\mathrm{f}$ electrons respectively, and

$$
\begin{aligned}
& \left|E, \epsilon^{\prime}, \epsilon\right\rangle=\frac{1}{\sqrt{N(N-1)}} \sum_{\nu, \nu^{\prime}, \nu \neq \nu^{\prime}} c_{E \nu^{\prime}}^{\dagger} c_{\epsilon^{\prime}, \nu^{\prime}} c_{\epsilon \nu} f_{\nu}^{\dagger}|0\rangle \\
& \left|E, \epsilon^{\prime}, \epsilon^{\prime \prime}, \epsilon\right\rangle=\frac{1}{\sqrt{N(N-1)(N-2)}} \sum_{\substack{\nu, \nu^{\prime}, \nu^{\prime \prime} \\
\nu^{\prime} \neq \nu \\
\nu^{\prime \prime} \neq \nu, \nu^{\prime}}} c_{E \nu^{\prime}}^{\dagger} c_{\epsilon^{\prime} \nu^{\prime}} c_{\epsilon^{\prime \prime} \nu^{\prime \prime}} f_{\nu^{\prime \prime}}^{\dagger} c_{\epsilon \nu} f_{\nu}^{\dagger}|0\rangle
\end{aligned}
$$


which are the $\mathrm{f}^{1}$ and $\mathrm{f}^{2}$ states, with one conduction-electron-hole pair included, to the order of $1 / N$. Within this $(1 / N)$-order basis we may write the full wavefunction

$$
\begin{gathered}
\left|\psi_{s}\right\rangle=\sum_{\epsilon} \alpha(\epsilon)|\epsilon\rangle+\sum_{\substack{\epsilon, \epsilon^{\prime} \\
\epsilon<\epsilon^{\prime}}} \beta\left(\epsilon, \epsilon^{\prime}\right)\left|\epsilon, \epsilon^{\prime}\right\rangle+\sum_{\epsilon, \epsilon^{\prime}, E} \gamma\left(E, \epsilon, \epsilon^{\prime}\right)\left|E, \epsilon, \epsilon^{\prime}\right\rangle \\
+\sum_{\substack{\epsilon, \epsilon^{\prime}, \epsilon^{\prime \prime}, E \\
\epsilon<\epsilon^{\prime}}} \delta\left(E, \epsilon^{\prime \prime}, \epsilon, \epsilon^{\prime}\right)\left|E, \epsilon^{\prime \prime}, \epsilon, \epsilon^{\prime}\right\rangle .
\end{gathered}
$$

The first two terms in this wavefunction refer to the lowest-order $f^{1}$ and $f^{2}$ components compensated by one- and two-hole operators with the same angular momentum. The third and fourth terms are simple extensions of these to include a single electron-hole pair (with the same angular momentum among the electron-hole pair members). The energy variables are restricted so as to be negative for hole operators and positive for electron operators.

Acting with the Hamiltonian on these states, equating terms with the same operator products, and eliminating the $f^{2}$ components leads to the coupled integral equations

$$
\left(\epsilon_{10}^{\mathrm{s}}-\epsilon\right) \alpha(\epsilon)=\Gamma \int_{-D}^{0} \mathrm{~d} \epsilon \frac{\alpha(\epsilon)+\alpha\left(\epsilon^{\prime}\right)}{\epsilon_{20}^{s}-\epsilon-\epsilon^{\prime}}+\frac{\Gamma^{2}}{N} \int_{-D}^{0} \mathrm{~d} \epsilon^{\prime} \int_{0}^{D} \mathrm{~d} E \frac{Q\left(E, \epsilon, \epsilon^{\prime}\right)+Q\left(E, \epsilon^{\prime}, \epsilon\right)}{\epsilon_{20}^{\mathrm{s}}-\epsilon-\epsilon^{\prime}}
$$

$$
d^{s}\left(E-\epsilon-\epsilon^{\prime}\right) Q\left(E, \epsilon, \epsilon^{\prime}\right)=\Gamma\left(\frac{\alpha(\epsilon)+\alpha\left(\epsilon^{\prime}\right)}{\epsilon_{20}^{\mathrm{s}}-\epsilon-\epsilon^{\prime}}\right)+\Gamma \int_{-D}^{0} \mathrm{~d} \epsilon^{\prime \prime} \frac{Q\left(E, \epsilon, \epsilon^{\prime \prime}\right)}{\epsilon_{20}^{\mathrm{s}}-\epsilon-\epsilon^{\prime}-\epsilon^{\prime \prime}+E}
$$

where

$d^{\mathrm{s}}\left(E-\epsilon-\epsilon^{\prime}\right)=\epsilon_{10}^{\mathrm{s}}+E-\epsilon-\epsilon^{\prime}-\Gamma \log \left[\left(\epsilon_{20}^{\mathrm{m}}+E-\epsilon-\epsilon^{\prime}+D\right) /\left(\epsilon_{20}^{\mathrm{m}}+E-\epsilon-\epsilon^{\prime}\right)\right]$

and

$$
Q\left(E, \epsilon, \epsilon^{\prime}\right) \equiv \gamma\left(E, \epsilon, \epsilon^{\prime}\right) / V[V \sqrt{(N-1)}] .
$$

The first of these is straightforwardly manipulated [13] to yield

$$
\delta E_{\mathrm{sin}}=-\int_{-D}^{0} \mathrm{~d} \epsilon \int_{-D}^{0} \mathrm{~d} \epsilon^{\prime} \int_{0}^{D} \mathrm{~d} E \frac{\alpha_{0}(\epsilon)+\alpha_{0}\left(\epsilon^{\prime}\right)}{\epsilon_{20}^{\mathrm{s}}-\epsilon-\epsilon^{\prime}} Q\left(E, \epsilon, \epsilon^{\prime}\right)
$$

with $\delta E_{\text {sin }}$ defined by $E^{s}=E_{0}^{\mathrm{s}}+(1 / N) \delta E_{\mathrm{sin}}, Q\left(E, \epsilon, \epsilon^{\prime}\right)$ determined by (21), and functions $\alpha_{0}(\epsilon)$ taken at leading order in $1 / N$.

\section{Analytic properties of the integral equations}

Both the singlet and magnetic states involve the solution of integral equations with very similar structure. These can be written rather simply, in terms of an operator $K(x)$ defined such that

$$
K(x) \mathrm{f}(x)=d(-x) f(x)-\Gamma \int_{-D}^{0} \mathrm{~d} \epsilon \frac{f(\epsilon)}{\epsilon_{20}^{\mathrm{s}}-\epsilon-x}
$$


so that for the magnetic state we are required to solve

$$
K\left(E-\epsilon+\epsilon_{20}^{\mathrm{m}}-\epsilon_{20}^{\mathrm{s}}\right) Q_{2}(E, \epsilon)=\Gamma /\left(\epsilon_{20}^{\mathrm{m}}-\epsilon\right)
$$

while for the singlet

$$
K\left(E-\epsilon-\epsilon^{\prime}\right) Q\left(E, \epsilon, \epsilon^{\prime}\right)=\Gamma\left[\left(\alpha_{0}(\epsilon)+\alpha_{0}\left(\epsilon^{\prime}\right) /\left(\epsilon_{20}^{\mathrm{s}}-\epsilon-\epsilon^{\prime}\right)\right]\right.
$$

The special feature of this kernel is that it involves the leading-order binding energy, which is itself determined from the condition $K(\epsilon) \alpha_{0}(\epsilon)=0$. Hence we have the task of solving separate inhomogeneous integral equations, knowing that in the low-energy limit we have to recover consistency with the homogeneous equation. As a result the functions $Q$ and $Q_{2}$ have to be singular in the limits $E-\epsilon-\epsilon^{\prime}=0$ and $E-\epsilon+\epsilon_{20}^{\mathrm{m}}-\epsilon_{20}^{\mathrm{s}}=0$ respectively. This singular behaviour can be extracted by multiplying (24) and (25) by $\alpha_{0}(\epsilon)$ and integrating over $\epsilon$ from $-D$ to zero after which we obtain for the singlet function $Q$

$$
Q\left(E, \epsilon, \epsilon^{\prime}\right)=\alpha_{0}\left(\epsilon^{\prime}\right) q(\epsilon) /(E-\epsilon)
$$

together with a correction regular in $E-\epsilon$, where

$$
q(\epsilon)=\int_{-D}^{0} \mathrm{~d} \epsilon^{\prime} \alpha_{0}\left(\epsilon^{\prime}\right)\left[\alpha_{0}(\epsilon)+\alpha_{0}\left(\epsilon^{\prime}\right)\right] /\left(\epsilon_{20}^{\mathrm{s}}-\epsilon-\epsilon^{\prime}\right)
$$

The quantity $E-\epsilon$ represents a boson-like energy, referring as it does to the energy of an electron-hole pair. This energy never becomes negative.

As far as the magnetic-state function is concerned, a similar analysis yields

$$
Q_{2}(E, \epsilon)=\frac{\alpha_{0}(\epsilon)}{E-\delta} \int_{-D}^{0} \mathrm{~d} \epsilon^{\prime} \frac{\alpha_{0}\left(\epsilon^{\prime}\right)}{\epsilon_{20}^{\mathrm{m}}-\epsilon^{\prime}}
$$

where $\delta=\epsilon_{20}^{s}-\epsilon_{20}^{\mathrm{m}}$. We note that the contribution from $Q_{2}$ to the magnetic-state binding energy involves integrating over negative values of $E-\delta$ as well as positive ones. A different kind of singularity arises in $Q_{2}(E, \epsilon)$ as $E-\epsilon$ vanishes, since $d^{\mathrm{m}}(E-\epsilon)$ itself vanishes in this limit. A solution can be found to leading logarithmic order by iterating the Neumann series. We find in this way that

$$
Q_{2}(E, \epsilon)=\frac{\Gamma}{d^{\mathrm{m}}(E-\epsilon)\left(\epsilon_{20}^{\mathrm{m}}-\epsilon\right)\left\{1+\left[\Gamma /\left(\epsilon_{20}^{\mathrm{m}}+\Gamma\right)\right] \log \left(E / E_{\mathrm{c}}\right)\right\}}
$$

where $E_{\mathrm{c}}$ is a cut-off of the order of $\Gamma$. The point of this relation is that the integral $\int_{-D}^{0} \mathrm{~d} \epsilon Q_{2}(E, \epsilon)$ converges to a finite limit in the low- $E$ region and that therefore numerical evaluation of $Q_{2}$ at very low energies, where the integral equation is not well behaved, is not problematic. 


\section{Numerical procedures}

The integral equations for $Q\left(\epsilon, \epsilon^{\prime}, E\right)$ and $Q_{Z}(\epsilon, E)$ must be treated specially because Neumann's series does not converge in the regions where the kernel of the integral equations are nearly the same as the kernel for the singlet equation. For both cases the kernel of the integral equation has the form

$$
K(x, y)=1 /(\alpha-x-y)
$$

where $x, y$ range from $-D$ to zero and $\alpha$ is always positive. We approximate this $K(x, y)$ with its Taylor-series expansion. Because $\alpha$ can be small, the Taylor series only converges for a limited range, so we actually expand $K(x, y)$ about several different points.

$$
K(x, y)=\sum_{i=1}^{N} \sum_{n=0}^{\infty} \frac{\left(y-y_{i}\right)^{n}}{\left(\alpha-y_{i}-x\right)^{n+1}} \Theta\left(y+r_{i}\right) \Theta\left[-\left(y+r_{i-1}\right)\right]
$$

where for a given $i, y_{i}$ is the value of $y$ about which the kernel is expanded and $r_{i}$ and $r_{i-1}$ are chosen so that the range in which the $\Theta$ functions do not vanish $y-y_{i}<\alpha-y_{i}$ and the series converges. This kernel has the form

$$
K(x, y)=\sum_{i=1}^{N} \sum_{n=0}^{\infty} X_{n i}(x) Y_{n i}(y)
$$

We then define $\alpha_{n i}=\int_{-D}^{0} \mathrm{dy} Q(y) Y_{n i}(y)$, and find after substituting into the integral equation

$$
f(x) Q(x)=b(x)+\int_{-D}^{0} \mathrm{~d} y K(x, y) Q(y)
$$

the linear system of equations

$$
\alpha_{n i}=b_{n i}+\sum_{n i, m j} \alpha_{m j} A_{n i}^{m j}
$$

where

$$
\begin{aligned}
& b_{n i}=\int_{-D}^{0} \mathrm{~d} x \frac{Y_{n i}(x) b(x)}{f(x)} \\
& A_{n i}^{m j}=\int_{-D}^{0} \mathrm{~d} x \frac{X_{m j}(x) Y_{n i}(x)}{f(x)}
\end{aligned}
$$

We then solve the linear system for $\alpha_{n i}$ (with an appropriate limitation on the $n$ summation) and obtain

$$
Q(x)=\frac{1}{f(x)}\left(b(x)+\sum_{n i} \alpha_{n i} X_{n i}(x)\right)
$$

We note that in the case of the singlet $1 / N$ calculation $\alpha=\epsilon_{20}^{\mathrm{s}}+E-\epsilon$. We used an LU decomposition method [16] for solving the linear system (33). This method decomposes the matrix $\delta_{n i}^{m j}-A_{n i}^{m j}$ into the product of a lower triangular matrix and an upper triangular 
matrix and uses the fact that the solutions of triangular-matrix equations are trivial. For this method the change of variables to $E-\epsilon$ and $E+\epsilon$ resulted in a very large increase in computational efficiency. This is because the kernel of equation (21), and hence $A_{n i}^{m j}$, only depends upon $E-\epsilon$ and the matrix $\delta_{n i}^{m j}-A_{n i}^{m j}$ must then be decomposed a number of times equal to the number $N_{\mathrm{s}}$ of sampled points, rather than $N_{\mathrm{s}}^{2}$ times. Once the matrix has been decomposed, the linear system (33) may be quickly solved for each of the sets $b_{n i}$. This change of variables has the additional advantage that the form of the transformation causes the contribution of the singular region, when the kernel for $Q\left(\epsilon, \epsilon^{\prime}, E\right)$ is very near the kernel for the leading-order wavefunction, to become finite. In the case of the magnetic equation $\alpha=\epsilon_{20}^{\mathrm{m}}+E$. The above singularity in these equations we treat as a principal part integration by cutting off the calculation $10^{-3} \mathrm{\Gamma}$ to either side of the pole.

\section{Results}

Before examining the numerical results we note that a very useful qualitative guide to the solution of the problem can be gained from looking at the equations for $Q$ and $Q_{2}$ at large energy arguments. We find that in this limit

$$
Q_{2}(\epsilon, E) \simeq \frac{\Gamma}{d^{\mathrm{m}}(E-\epsilon)\left(\epsilon_{20}^{\mathrm{m}}-\epsilon\right)}=Q_{1}(\epsilon, E)
$$

which, after substituting in (13), yields

$$
\left(1+\frac{\Gamma}{\epsilon_{20}^{\mathrm{m}}}-\frac{\Gamma}{\left(\epsilon_{20}^{\mathrm{m}}+D\right)}\right) \delta E_{\mathrm{mag}}=-\Gamma \int_{-D}^{0} \mathrm{~d} \epsilon \int_{0}^{D} \mathrm{~d} E \frac{2 \Gamma}{\left(\epsilon_{20}^{\mathrm{m}}-\epsilon\right)^{2} d^{\mathrm{m}}(E-\epsilon)} .
$$

This can be further approximated by dropping the logarithmic term in $d^{\mathrm{m}}(E-e)$

$$
\delta E_{\mathrm{mag}}=\left[-2 \Gamma^{2} /\left(\epsilon_{20}^{\mathrm{m}}+\Gamma\right)\right] \log \left(D / E_{\mathrm{c}}\right)
$$

where $E_{\mathrm{c}}$ is a cut-off of the order of $\Gamma$. Similarly, for the singlet state we find that

$$
Q\left(\epsilon, \epsilon^{\prime}, E\right)=\frac{\Gamma\left[\alpha_{0}(\epsilon)+\alpha_{0}\left(\epsilon^{\prime}\right)\right]}{d^{s}\left(E-\epsilon-\epsilon^{\prime}\right)\left(\epsilon_{20}^{s}-\epsilon-\epsilon^{\prime}\right)} .
$$

Inserting this expression into the right-hand side of (22) results in

$$
\delta E_{\mathrm{sin}}=-\Gamma \int_{-D}^{0} \mathrm{~d} \epsilon \int_{-D}^{0} \mathrm{~d} \epsilon^{\prime} \int_{0}^{D} \mathrm{~d} E \frac{\left[\alpha_{0}(\epsilon)+\alpha_{0}\left(\epsilon^{\prime}\right)\right]^{2}}{\left(\epsilon_{20}^{s}-\epsilon-\epsilon^{\prime}\right)^{2} d^{s}\left(E-\epsilon-\epsilon^{\prime}\right)}
$$

which can be further approximated by integrating over $E$ and dropping the logarithmic dependence on $\epsilon$ and $\epsilon^{\prime}$ in favour of the extremely peaked energy dependence of $\alpha_{0}$. Then we apply the identity

$$
\int_{-D}^{0} \mathrm{~d} \epsilon \int_{-D}^{0} \mathrm{~d} \epsilon^{\prime} \frac{\left[\alpha_{0}(\epsilon)+\alpha_{0}\left(\epsilon^{\prime}\right)\right]^{2}}{\left(\epsilon_{20}^{\mathrm{s}}-\epsilon-\epsilon^{\prime}\right)^{2}}=2\left(n_{\mathrm{f}}-1\right)
$$

which can be easily obtained from the ground-state wavefunction using the definitions of the valence $n_{\mathrm{f}}=\left\langle\psi_{0}\left|\sum_{\mathrm{m}} f_{\mathrm{m}}^{\dagger} f_{\mathrm{m}}\right| \psi_{0}\right\rangle$. We find that to leading logarithmic order in the bandwidth

$$
\delta E_{\sin }=2 \Gamma\left(n_{\mathrm{f}}-1\right) \log \left(D / E_{\mathrm{c}}\right)
$$


where $E_{\mathrm{c}}$ is again an unspecified cut-off of the order of $\Gamma$. While the above estimates are mainly of qualitative use, we shall see that they allow a straightforward semi-quantitative representation of the $1 / N$ corrections. In figure 1 , we show the numerical solutions for $\delta E_{\text {mag }}$ and $\delta E_{\text {sin }}$ as a function of $E_{21}$ for two representative bandwidths $D=50$ and $D=200$ together with the asymptotic estimates for these quantities obtained from (37) and (40). We note that the asymptotic results for these energies show the same qualitative features throughout the valence regime that for $D / \Gamma=50$ is covered by taking $E_{21}$ values from $0\left(n_{\mathrm{f}}^{0}=1.34\right)$ to $-7\left(n_{\mathrm{f}}^{0}=1.94\right)$ and for $D / \Gamma=200$ is covered by taking $E_{21}$ values from $0\left(n_{\mathrm{f}}^{0}=1.21\right)$ to $-8\left(n_{\mathrm{f}}^{0}=1.96\right)$. The agreement is better for the magnetic states since the corrections due to the integral equations only apply to the contribution of $Q_{2}$ in (13). Furthermore we find that the separation $\delta E_{\operatorname{mag}}-\delta E_{\sin }$ is small and positive in the $\mathrm{f}^{1}$ regime $\left(E_{21} \simeq 0\right)$, increases in the centre of the valence regime $\left(E_{21} \simeq-3\right)$, and changes sign as we approach the $\mathrm{f}^{2}$ limit $\left(E_{21} \simeq-5\right)$. The change of sign between the $\mathrm{f}^{1}$ and $\mathrm{f}^{2}$ limits can be understood by studying the perturbative scaling calculations of the Kondo temperature in those limits, since we expect the singlet-magnetic energy difference to correspond to this energy scale in the local moment limits. Taking $\tilde{T}$ and $T^{*}$ as the renormalized ionic separations in the $f^{1}$ and $f^{2}$ limits respectively, we find that in the $f^{2}$ limit $T_{\mathrm{K}}=D_{0}\left(\Gamma / D_{0}\right)^{1 / N}(\tilde{T} / D)^{2 / N} \exp \left(E_{21} / \Gamma\right)[8]$, so the $1 / N$ expansion of this quantity yields a negative $\log (D)$ contribution. On the other hand the $\mathrm{f}^{1}$ limit has a Kondo temperature $T_{\mathrm{K}}=D(\Gamma / D)^{1 / N}\left(T^{*} / D\right)^{2(1-2 / N)} \exp \left(-E_{21} / \Gamma\right)$ for which the $1 / N$ term has a positive $\log D$ contribution. Hence a change of sign in the intermediate-valence regime is to be expected.

Turning to a more detailed examination of the numerical results we show in figure 2 the $1 / N$ corrections to the singlet-magnetic energy difference, together with the leading-order result for $D=50$ and $D=200$. Again, the numerical results for the $1 / N$ corrections are consistent with the approximations, showing an increase on leaving the $\mathrm{f}^{1}$ regime, a maximum in the mixed-valence regime, followed by a reduction, leading to a change of sign in the $\mathrm{f}^{2}$ limit, again turning over and vanishing asymptotically in the extreme $\mathrm{f}^{2}$ limit.

Combining the leading- and next-leading-order results, we show in figure 3 the full singlet-magnetic separation for $N=6,8,14$, and $\infty$. We note that the maximum positions are shifted over to smaller values of $E_{21}$, and that the values of $E_{\mathrm{m}}-E_{\mathrm{s}}$ are reduced in the $\mathrm{f}^{2}$ limit from the infinite degeneracy limit, consistent with scaling theory. The central result, however, concerns the actual value of the maximum singlet-magnetic splitting that results from the next-leading $1 / \mathrm{N}$ calculation. Even for the smallest degeneracies the increase in peak height is no more than $20 \%$ over the zeroth-order calculation. This is important, since one might expect the extra logarithmic (in $D$ ) contributions that result from the extra particle-hole states to completely dominate over the small energy scale that appears at leading order. This does not happen, because of a cancellation of part of this logarithmic term between the singlet- and magnetic-state energies. The small energy scale therefore appears to be robust, with a maximum value of $\sim 12 \%$ of the hybridization width for the chosen bandwidth values. Since, as we have seen, the bandwidth dependence of the $1 / N$ corrections is essentially logarithmic we do not expect a strong dependence of the peak value on bandwidth, for realistic values.

While this constitutes the essential physical result of our calculation, the abundance of parameters in the Anderson model makes it desirable to represent the results in terms of physical parameters. For example a central feature of the $f^{0}-f^{1}$ model is the functional dependence of the susceptibility on the valence [14]. This facilitates, for the $\mathrm{f}^{0}-\mathrm{f}^{1}$ model, a detailed comparison between the $1 / N$ method and exact results [14]. For our model, following Evans and Gehring [10], we would expect the susceptibility to be inversely 

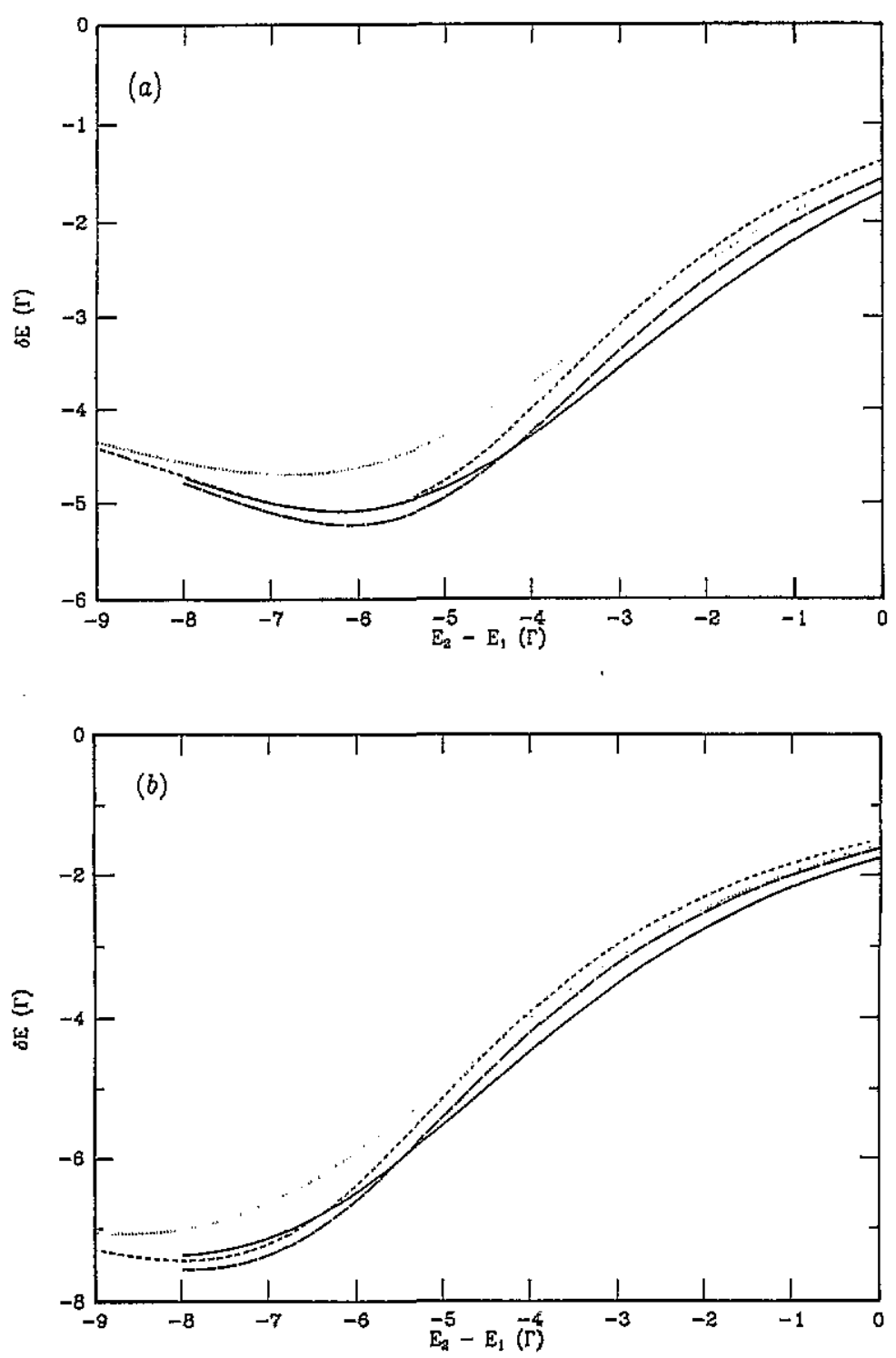

Figure 1. The $1 / N$ corrections to the singlet (full curve) and magnetic (long dashes) energies, $\delta E / N=E-E_{0}$, as a function of ionic splitting for $(a) D=50$ and $(b) D=200$. Also shown in each case are the approximations (37) and (40) for the $1 / N$ corrections to the singlet-state (dots) and magnetic-state (short dashes) energies.

proportional to the singlet-magnetic splitting. Therefore the universal properties of the model would be best expressed as a plot of the splitting versus total (leading- and nextleading-order valence). We show this in figure 4 . We note that the maximum of the splitting is shifted to slightly higher values of the valence rather than to the centre, as is found in variational [10] and NCA treatments of the minimal-degeneracy model. We ascribe this, in part, following Evans and Gehring's observation of the same effect in their variational calculation [10], to the degeneracy of the $\mathrm{f}^{2}$ states. In addition we expect that our inclusion of the $f^{2}$ particle-hole states enhances this effect. 

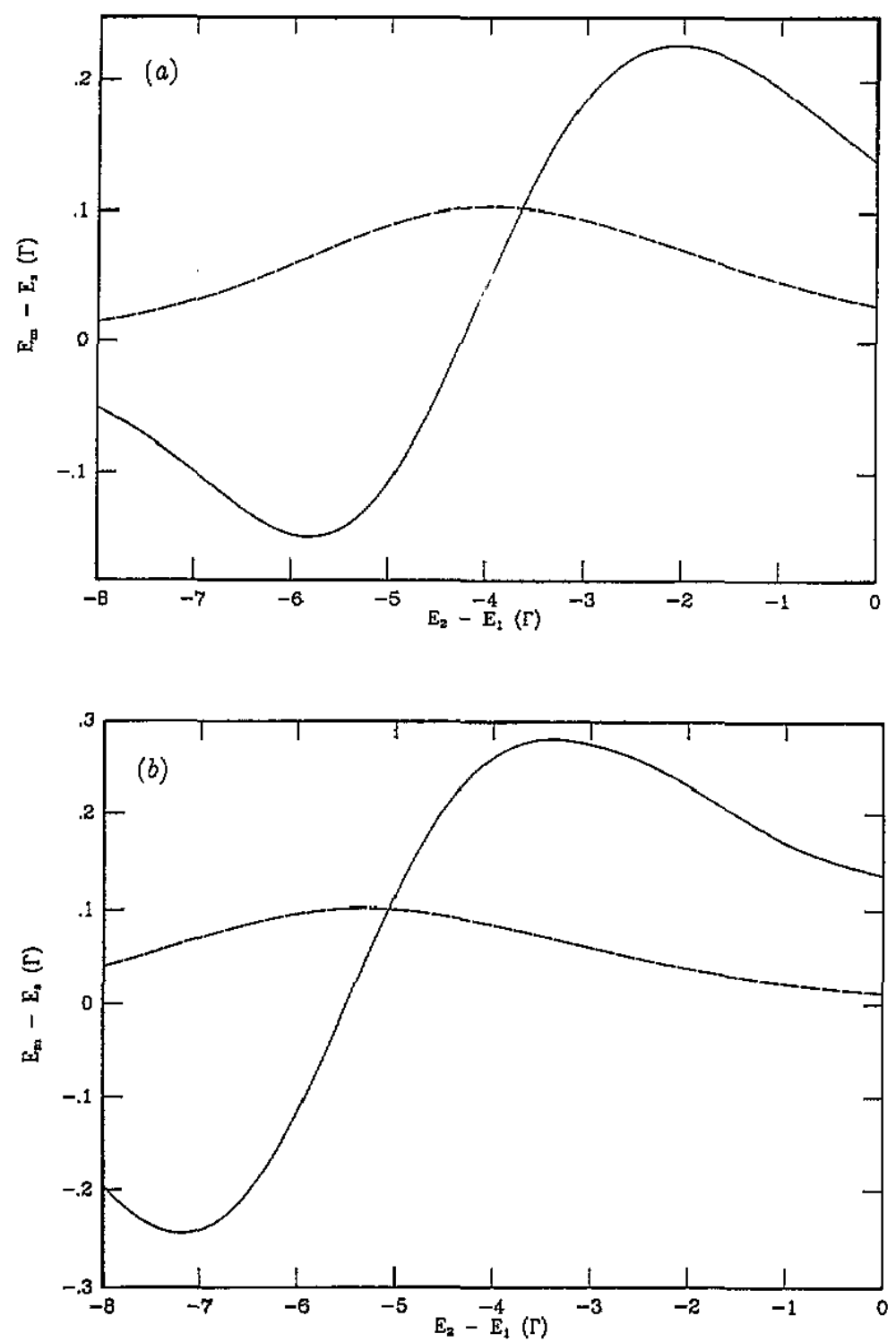

Figure 2. The $1 / N$ corrections to the singlet-magnetic energy difference (full curve) as a function of ionic separation for (a) $D=50$ and $(b) D=200$. Also shown are the leading-order results (dashed curve).

Another issue we have to be concerned about is the behaviour of the singlet-magnetic separation close to the $\mathrm{f}^{2}$ limit. From figure 4 it is apparent that the binding energy is reduced below the leading order value, in an analogous manner to the behaviour close to $n_{\mathrm{f}}=1$ in the $\mathrm{f}^{0}-\mathrm{f}^{1}$ model [14]. We have problems in picking out the behaviour close to $n_{\mathrm{f}}=2$ in our calculation, because of the tiny energy scales involved in this limit. However, we can see in figure 5 that the total valence itself, as a function of $E_{21}$, approaches the $n_{\mathrm{f}}=2$ limit more slowly than the leading-order calculation on account of the finite $\Gamma / E_{21}$ corrections that are obtained at the $1 / N$ level [8].

Finally, in figure 6 we show the charge susceptibility $\chi_{\mathrm{ch}}=-\partial n_{\mathrm{f}} / \partial E_{21}$ as a function 

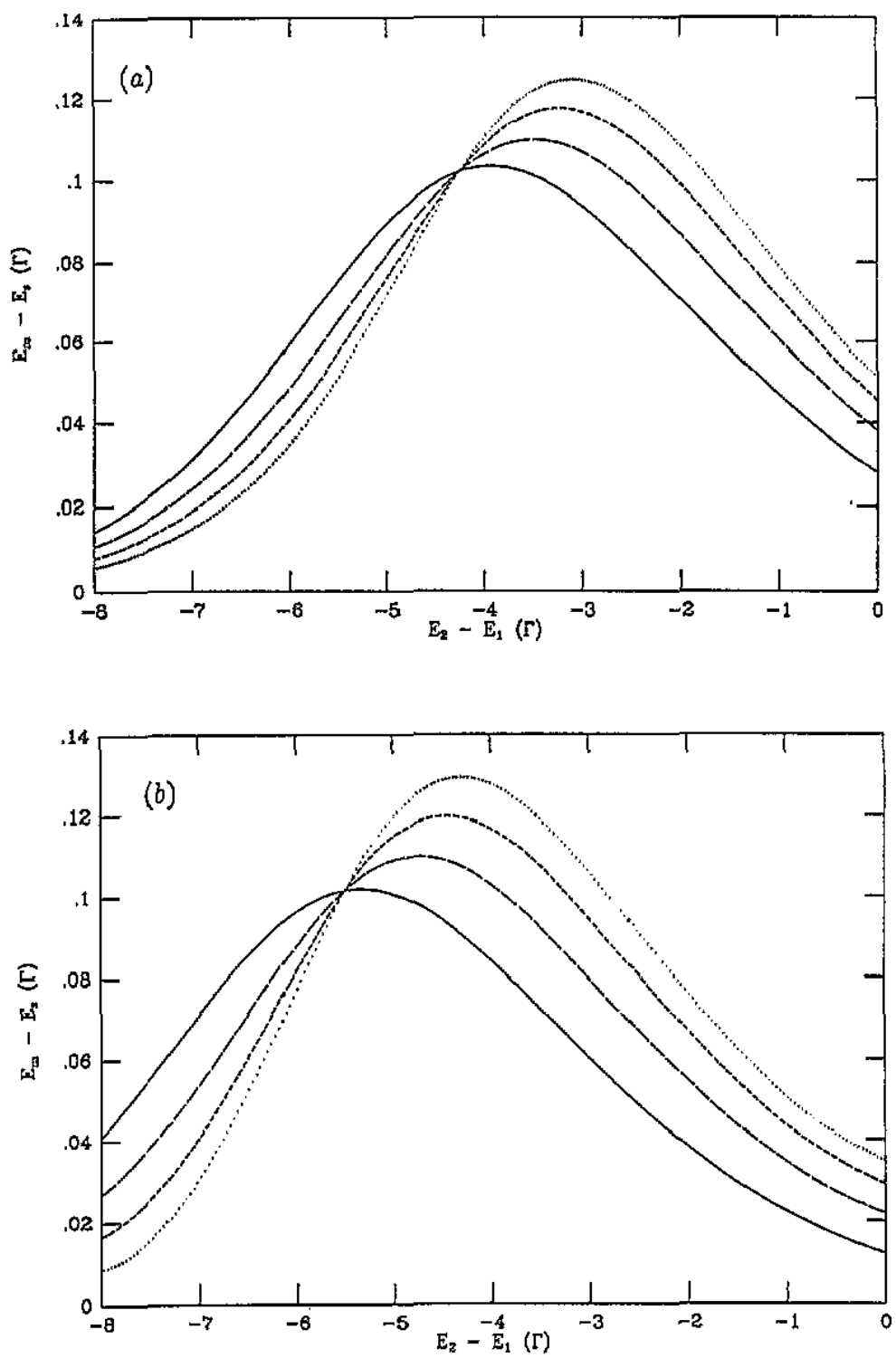

Figure 3. Total separation between the magnetic- and singlet-state energies as a function of ionic separation to next-leading order in $1 / N$ for $N=\infty$ (full curve), $N=14$ (long dashes), $N=8$ (short dashes), and $N=6$ (dots), for (a) $D=50$ and (b) $D=200$.

of the total valence. We find a maximum value of approximately 0.1 , with only a slight deviation of the peak height from the $1 / N$ corrections. We note that the peak position is shifted to smaller values. The results are consistent with the estimates by Evans and Gehring [10] of this quantity and confirm that for the model under study, the spin susceptibility of approximately $1 /\left(E_{\mathrm{m}}-E_{\mathrm{s}}\right)$ is at least two orders of magnitude larger than the charge susceptibility. 

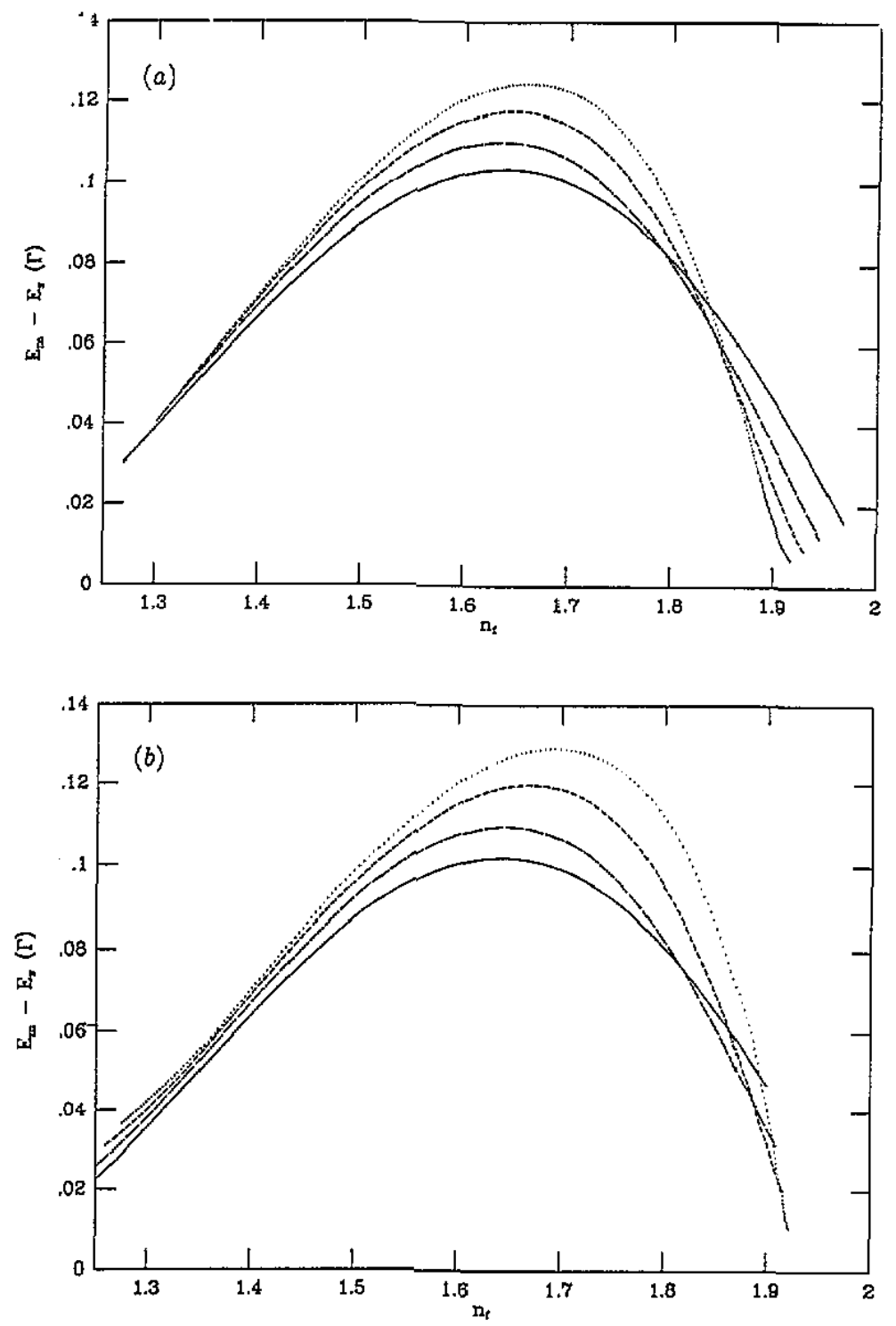

Figure 4. Total separation betwen the magnetic- and singlet-state energies as a function of total valence for $N=\infty$ (full curve), $N=14$ (long dashes), $N=8$ (short dashes), and $N=6$ (dots), for (a) $D=50$ and (b) $D=200$.

\section{Summary and conclusions}

In this paper we have systematically studied the ground-state energy and related properties such as the valence and charge susceptibility of an $\mathrm{N}$-fold-degenerate Anderson model fluctuating between $\mathrm{f}^{1}$ and $\mathrm{f}^{2}$ configurations to next-leading order in the $1 / N$ expansion. Our motivation was to see whether the essentially unique features of the problem that appear at leading order [8] (or in related variational treatments of less degenerate models $[7,10]$ ) persist in the presence of the full set of particle-hole excitations. For the cerium $f^{0}-f^{1}$ problem it is known that these excitations affect certain individual properties, such as the 


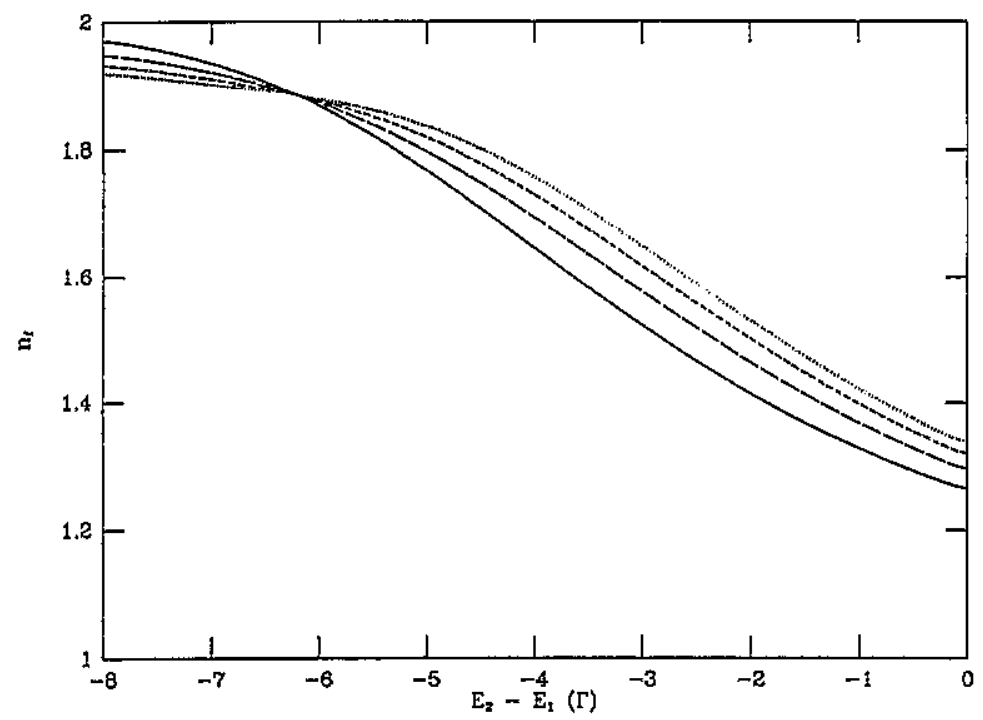

Figure 5. Total valence as a function of ionic separation for $N=\infty$ (full curve), $N=14$ (long dashes), $N=8$ (short dashes), and $N=6$ (dots), for $D=50$.

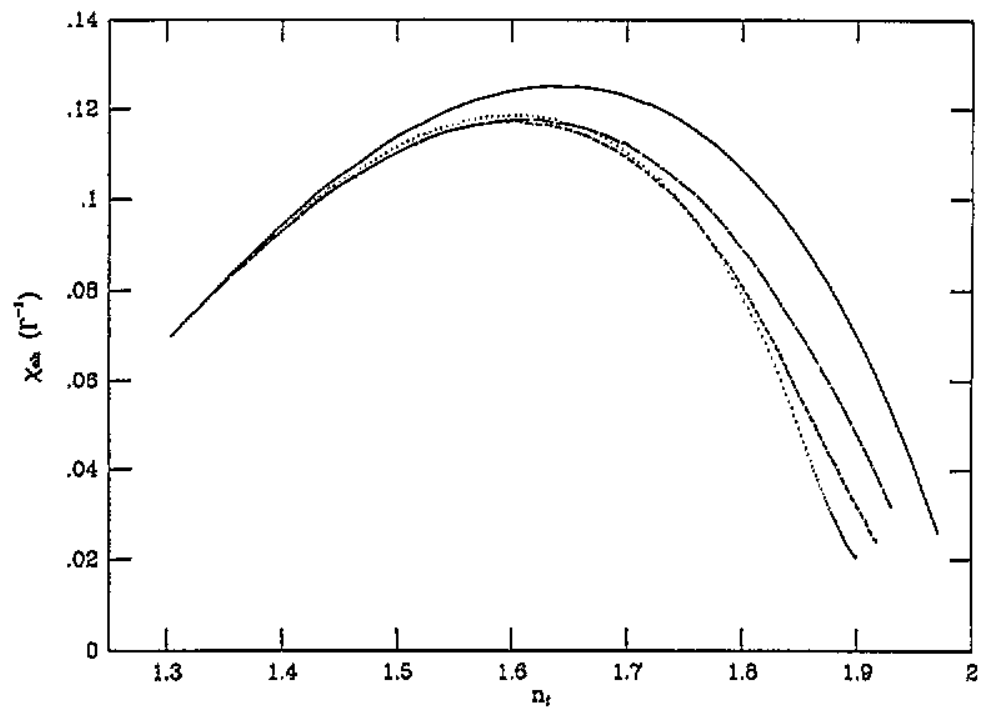

Figure 6. Charge susceptibility as a function of total valence for $N=\infty$ (full curve), $N=14$ (long dashes), $N=8$ (short dashes), and $N=6$ (dots), for $D=50$.

ground-state energy, but not universal features. We formulated the problem in the restricted basis method of Gunnarsson and Schönhammer [15] and derived integral equations for the singlet- and magnetic-state energies, which were solved numerically.

We found that the singlet-magnetic energy separation was little changed from its leadingorder value, despite large individual $1 / N$ corrections to the singlet- and magnetic-state energies. These $1 / N$ corrections changed both sign and curvature through the full valence 
region, consistent with earlier scaling results in the local moment limits. As a function of the total valence the peak in the singlet-magnetic separation was shifted to higher values of the valence, on account of the higher degeneracy of the $f^{2}$ state. The valence approached the $\mathrm{f}^{2}$ limit more slowly than the leading-order result because of the inclusion of nonanalytic corrections known from scaling theory. The charge susceptibility remained small, being hardly affected by the $1 / N$ corrections, and even in the middle of the valence regime remained roughly two orders of magnitude less than the spin susceptibility.

Overall, the results of our systematic $1 / N$ calculation confirm the existence of a small energy scale $(\sim 0.1 \Gamma$ ) separating the many-body singlet ground state from higher-lying magnetic states, as well as the relative unimportance of charge fluctuations compared with spin fluctuations in impurity models where both atomic configurations are degenerate. These results should be of importance in assessing the ease with which intersite effects can stabilize magnetic ordering in concentrated actinide alloys and compounds.

\section{Appendix}

It may be argued that the result that the singlet state (19) has lower energy than the magnetic state (8) is due to the fact that if treated as variational wavefunctions the singlet state has a larger parameter space than the magnetic states. It is indeed the case that the lower energy of the singlet state is due to the extra energy which is gained via hybridization with the larger number of non-interacting basis states that are included in (19). In this appendix we present two arguments that, at least in the framework of the formal $1 / N$ expansion, the reduced parameter space of the magnetic states is a necessary result of the form of the interactions rather than an arbitrary one.

The basic idea is that any conduction-hole-f-electron pair term, for which the conduction hole and the $f$ electron do not have the same magnetic index, cannot be either created or destroyed by the mixing term of the Hamiltonian (1). Any basis state that contains such unscreened terms may only couple to other basis states via the destruction or creation of other screened pairs, so that the effect of the unscreeened pairs is simply the addition of the single-particle energies to the energy of the basis state. The lowest such state will be obtained by choosing the energy of the conduction hole to be at the Fermi surface. The conduction-hole-f-electron term may then be replaced in the definition of the basis state by a single $f$ electron operator and the magnetic-state definition (8) results.

A more concrete description of this may be obtained by constructing magnetic states with the same size parameter space as the singlet states. This can easily be done to leading order by using the states defined in section $2.1,\left|\epsilon^{(i)}\right\rangle(14)$ and $\left|\epsilon^{\prime}, \epsilon^{(i)}\right\rangle(15)$ :

$$
\left|\Psi^{(i)}\right\rangle=\sum_{\epsilon} \alpha^{(i)}(\epsilon)\left|\epsilon^{(i)}\right\rangle+\sum_{\epsilon, \epsilon^{\prime}} \beta^{(i)}\left(\epsilon, \epsilon^{\prime}\right)\left|\epsilon^{\prime}, \epsilon^{(i)}\right\rangle
$$

By acting on this wavefunction with the Hamiltonian we obtain

$$
\left\{\epsilon_{10}^{\mathrm{m}}-\epsilon-\Gamma \log \left[\left(\epsilon_{20}^{\mathrm{m}}-\epsilon+D\right) /\left(\epsilon_{20}^{\mathrm{m}}-\epsilon\right)\right]\right\} \alpha^{(i)}(\epsilon)=0
$$

and we note that this equation cannot give us the form of $\alpha^{(i)}$. In fact, the proper interpretation of (A2) is that the state (A1) is really just a superposition of eigenstates (at leading order in $1 / N$ )

$$
\left|\Psi^{(i)}(\epsilon)\right\rangle=\alpha^{(i)}(\epsilon)\left|\epsilon^{(i)}\right\rangle+\sum_{\epsilon^{\prime}} \beta^{(i)}\left(\epsilon ; \epsilon^{\prime}\right)\left|\epsilon^{\prime}, \epsilon^{(i)}\right\rangle
$$


where each of the eigenstates (A3) have energy $E_{0}^{\mathrm{m}}-\epsilon$ where $E_{0}^{\mathrm{m}}$ is the normal leadingorder magnetic state energy and $\epsilon$ is (as always) negative. Here again the lowest of the 'magnetic' states gives equivalent results to the leading-order analysis in section 2.1.

When we examine the validity of these results to next-leading order in $1 / \mathrm{N}$ a complication arises. Using the language of the first argument presented above, when there are several conduction-hole-f-electron pairs present, and if one or more of them are unscreened, it may happen that terms such as $c_{\epsilon \nu} f_{\nu}^{\dagger} c_{\epsilon^{\prime} \nu} f_{\mu}^{\dagger}|0\rangle$ occur. In such a term it is ambiguous whether the hole with energy $\epsilon$ or $\epsilon^{\prime}$ is paired with the f electron in channel $\nu$. This ambiguity leads to a $(1 / N)$-order mixing between each of the magnetic states with different energies (A3). While all of the off-diagonal matrix elements of the Hamiltonian to leading order are negative, these additional $1 / N$ terms, $\left\langle\epsilon^{(i)}|H| \epsilon, \epsilon^{\prime(l)}\right\rangle$, are positive. We shall see that when treated in the $1 / N$ expansion, these positive matrix elements lead to an increase of the magnetic state energy with respect to the magnetic state energy considered in the text.

In a completely variational approach the opposite is the case. Because the leading-order magnetic-state energy obtained in the text is exactly equal to that obtained variationally by restricting the basis states for the wavefunction to states of the form $\left|0^{(i)}\right\rangle$ and $\left|\epsilon, 0^{(i)}\right\rangle$, and because expanding the solution space in a variational calculation can only reduce the energy, we see that the magnetic energy is lowered despite the positive mixing terms. Even so, because the additional off diagonal matrix elements are positive, and because they are of order $1 / N$, the energy reduction will be quite small. In particular, the magnetic energy will remain larger than the singlet energy, because in that case the equivalent terms are negative and of order unity. While the $1 / N$ expansion technique and variational calculations lead to different solutions, the central result-that the singlet state is lower than the magnetic state-holds for both approaches.

We turn now to the evaluation of the $1 / N$ corrections to (A1). We must include the $\mathrm{f}^{1}$ basis states

$$
\begin{aligned}
& \left|E, \epsilon^{\prime}, \epsilon^{(i)}\right\rangle=\frac{1}{\sqrt{N-1}} \sum_{\nu} \sum_{\mu \neq \nu} c_{E \mu}^{\dagger} c_{\varepsilon^{\prime} \mu} C_{\nu}^{(i)} c_{\epsilon \nu} f_{\nu}^{\dagger}|0\rangle \\
& \left|\epsilon^{\prime},(E, \epsilon)^{(i)}\right\rangle=\frac{1}{\sqrt{N-1}} \sum_{\nu} \sum_{\mu \neq \nu} c_{\epsilon^{\prime} \mu} f_{\mu}^{\dagger} C_{\nu}^{(i)} c_{E \nu}^{\dagger} c_{\epsilon \nu}|0\rangle
\end{aligned}
$$

and the $\mathrm{f}^{2}$ basis states

$$
\begin{aligned}
& \left|E, \epsilon^{\prime}, \epsilon^{\prime \prime}, \epsilon^{(i)}\right\rangle=\frac{1}{(N-1)} \sum_{\nu} \sum_{\mu \neq \nu, \mu^{\prime} \neq \nu} c_{E \mu}^{\dagger} c_{\epsilon^{\prime} \mu} c_{\epsilon \mu^{\prime}} f_{\mu^{\prime}}^{\dagger} C_{\nu}^{(i)} c_{\epsilon \nu} f_{\nu}^{\dagger}|0\rangle \\
& \left|\epsilon^{\prime}, \epsilon^{\prime \prime},(E, \epsilon)^{(i)}\right\rangle=\frac{1}{(N-1)} \sum_{\nu} \sum_{\mu \neq \nu, \mu^{\prime} \neq \nu} c_{\epsilon^{\prime} \mu} f_{\mu}^{\dagger} c_{\epsilon^{\prime \prime} \mu^{\prime}} f_{\mu^{\prime}}^{\dagger} C_{\nu}^{(i)} c_{E \nu}^{\dagger} c_{\epsilon \nu}|0\rangle
\end{aligned}
$$

where in the second of these the energy variables $\epsilon^{\prime}$ and $\epsilon^{\prime \prime}$ are restricted to $\epsilon^{\prime} \leqslant \epsilon^{\prime \prime}$. These states are the straightforward generalizations of (6) and (7). We then apply the timeindependent Schrödinger equation to the most general wavefunction with the above basis states and obtain

$$
d^{\mathrm{m}}(-\epsilon) \alpha^{(i)}(\epsilon)=(\Gamma / N) \sum_{E, \epsilon^{\prime}} \frac{Q_{1}\left(E, \epsilon^{\prime}, \epsilon\right)+Q_{2}\left(E, \epsilon^{\prime}, \epsilon\right)}{\epsilon_{20}^{\mathrm{m}}-\epsilon-\epsilon^{\prime}}-(\Gamma / N) \sum_{\epsilon^{\prime}} \frac{\alpha^{(i)}\left(\epsilon^{\prime}\right)}{\epsilon_{20}^{\mathrm{m}}-\epsilon-\epsilon^{\prime}}
$$


where $Q_{1}$ and $Q_{2}$ are given by

$$
\begin{aligned}
& d^{\mathrm{m}}\left(E-\epsilon-\epsilon^{\prime}\right) Q_{1}\left(E, \epsilon^{\prime}, \epsilon\right)=\alpha^{(i)}(\epsilon) /\left(\epsilon_{20}^{\mathrm{m}}-\epsilon-\epsilon^{\prime}\right) \\
& d^{\mathrm{m}}\left(E-\epsilon-\epsilon^{\prime}\right) Q_{2}\left(E, \epsilon^{\prime}, \epsilon\right)=\frac{\alpha^{(i)}(\epsilon)}{\epsilon_{20}^{\mathrm{m}}-\epsilon-\epsilon^{\prime}}+\Gamma \int_{-D}^{0} \mathrm{~d} \epsilon^{\prime \prime} \frac{Q_{2}\left(E, \epsilon^{\prime \prime}, \epsilon\right)}{\epsilon_{20}^{\mathrm{m}}+E-\epsilon-\epsilon^{\prime}-\epsilon^{\prime \prime}} .
\end{aligned}
$$

The above set of equations are a coupled set of integral equations that determine the energy of the magnetic states.

In order to solve these equations using the $1 / N$ expansion one must first solve the equations to leading order. As discussed previously, the leading-order solution separates into different eigenstates for each $\epsilon$. Because we are working at zero temperature this requires that the leading-order magnetic-state equation is simply the lowest of these states. Hence, $\alpha_{0}^{(i)}(\epsilon)=\alpha_{0} \delta(\epsilon)$ where $\delta(\epsilon)$ is the Dirac delta function, and $\alpha_{0}$ is a constant determined by the normalization and is precisely the same quantity as in the formulation in section 2.1. Here the reason that the $1 / N$ and variational techniques lead to different results is apparent. In both cases, the same set of basis elements is used to each order in $1 / N$. However, in the $1 / N$ expansion the wavefunction in the higher-order space is determined by the lower-order wavefunction, while in a variational approach one is faced with the problem of coupled many-dimensional integral equations.

The assumption that $\alpha_{0}^{(i)}=\alpha_{0} \delta(\epsilon)$ drastically simplifies equations (A8) and (A9). After expanding to next-leading-order in $1 / N$ we obtain

$$
\begin{aligned}
\left(1+\frac{\Gamma}{\epsilon_{20}^{\mathrm{m}}}-\right. & \left.\frac{\Gamma}{\epsilon_{20}^{\mathrm{m}}+D}\right) \delta E_{\mathrm{mag}} \\
& =\Gamma \log \left(\frac{\epsilon_{20}^{\mathrm{m}}+D}{\epsilon_{20}^{\mathrm{m}}}\right)-\Gamma \int_{-D}^{0} \mathrm{~d} \epsilon \int_{-D}^{0} \mathrm{~d} E \frac{Q_{1}(\epsilon, E)+Q_{2}(\epsilon, E)}{\epsilon_{20}^{\mathrm{m}}-\epsilon}
\end{aligned}
$$

where, in this case, $Q_{1}$ and $Q_{2}$ are given by (11) and (12). This differs from the earlier result only in the first term on the right-hand side. This term has the effect of raising the energy correction of the magnetic state by a value $\epsilon_{20}^{\mathrm{m}} \epsilon_{10}^{\mathrm{m}} / \Gamma\left(\epsilon_{20}^{\mathrm{m}}+\Gamma\right)$, which vanishes in both integer-valent limits.

Thus, we have shown in this appendix that to next-leading-order in $1 / N$ such generalized magnetic states, with parameter spaces that are the same as for the singlet state, have in fact, energies higher to next-leading-order in $1 / N$ than those of the magnetic states considered in the text.

\section{References}

[1] Mazaferto J, Balseiro C A and Alascio B 1981 Phys. Rev. Lett. 47274

[2] Allub R, Ceva H and Alascio B 1984 Phys. Rev. B 293098

[3] Proetto C R, Aligia A A and Balseiro C A 1985 Phys. Lett. 107A 93

[4] Schlottmann P 1989 Phys. Rep. 1811

[5] Cox D L 1987 Phys. Rev. Lett. 591240

[6] Seaman C, Maple M B, Lee B W, Ghamaty S, Torikashvilli M S, Kang J S, Liu L Z, Allen J W and Cox D L 1991 Phys. Rev. Lett. 672882

[7] Yafet Y, Varma C M and Jones B A 1985 Phys. Rev, B 32360

[8] Read N, Dharmvir K, Rasul J W and Nunes D M 1986 J. Phys. C: Solid State Phys. 191597

[9] Nunes A C, Rasul J W and Gehring G 1986 J. Phys. C: Solid State Phys. 191017

[10] Evans S M and Gehring G 1989 J. Phys.: Condens. Matter 13095 
[11] Shimizu Y, Sakai O and Kasuya T 1990 Physica B 163401

[12] Saso T 1988 J. Magn. Magn. Mater. 76-77 176

[13] Rasul J W 1990 Phys, Rev. B 429996

[14] Rasul J W and Hewson A C 1984 J. Phys. C: Solid State Phys. 173337

[15] Gunnarsson O and Schönhammer K 1983 Phys. Rev. B 28 4315; 1985 Phys. Rev. B 314815

[16] Press W H, Flannery B P, Teukolsky S A and Vetterling W T 1988 Numerical Recipes (Cambridge: Cambridge University Press) 